\title{
Annihilation dynamics of reverse tilt domains in nematic liquid crystals
}

DOI:

10.1016/j.molliq.2020.113547

\section{Document Version}

Accepted author manuscript

Link to publication record in Manchester Research Explorer

\section{Citation for published version (APA):}

Shen, Y., \& Dierking, I. (2020). Annihilation dynamics of reverse tilt domains in nematic liquid crystals. Journal of Molecular Liquids, 313, 113547. https://doi.org/10.1016/j.molliq.2020.113547

\section{Published in:}

Journal of Molecular Liquids

\section{Citing this paper}

Please note that where the full-text provided on Manchester Research Explorer is the Author Accepted Manuscript or Proof version this may differ from the final Published version. If citing, it is advised that you check and use the publisher's definitive version.

\section{General rights}

Copyright and moral rights for the publications made accessible in the Research Explorer are retained by the authors and/or other copyright owners and it is a condition of accessing publications that users recognise and abide by the legal requirements associated with these rights.

\section{Takedown policy}

If you believe that this document breaches copyright please refer to the University of Manchester's Takedown Procedures [http://man.ac.uk/04Y6Bo] or contact uml.scholarlycommunications@manchester.ac.uk providing relevant details, so we can investigate your claim.

\section{OPEN ACCESS}




\title{
Annihilation Dynamics of Reverse Tilt Domains in Nematic Liquid Crystals
}

\author{
Yuan Shen, ${ }^{\mathrm{a}}$ and Ingo Dierking *a \\ ${ }^{a}$ Department of Physics \& Astronomy, School of Natural Sciences, University of Manchester, Oxford Road, Manchester M13 9PL, UK. \\ * Email: ingo.dierking@manchester.ac.uk
}

Reverse tilt domains are induced in a nematic liquid crystal confined in cells with near-zero pretilt angle homogeneous alignment. The domains emerge in the shape of "closed loops" and will annihilate over a time scale of seconds to minutes. The annihilation dynamics of single isolated domains as well as the coarsening dynamics of a number of domains are investigated in detail. The system maps onto the uniform Ising model and is characterized by a single length scale $L(t)$. The dynamical scaling laws previously proposed for coarsening dynamics of topological defects in symmetry breaking phase transitions are verified also for the electric field induced reverse tilt domains while varying external parameters, including electric field amplitude, frequency, temperature and cell gap.

Keywords: Liquid Crystals, Reverse tilt domains, Annihilation dynamics, Ising system, Scaling law

\section{Introduction}

During a symmetry breaking phase transition, defects are unavoidably generated as described by Kibble-Zurek mechanism [1-3], which dominate the subsequent equilibration process of the system. The equilibration process is characterized by the dynamic growth of domains of the ordered phase, which is called coarsening. Coarsening phenomena have been a critical topic in non-equilibrium statistical mechanics $[4,5]$, condensed matter physics [6] and cosmology [7] for decades. This is mostly due to the fascinating scaling dynamics that emerges in the growth of domains [8-11]. A typical example is the spinodal decomposition of binary mixtures for which a number of theories have been proposed to try to explain this scaling law [4, 10, 12]. Experimentally, due to their extremely small elastic constants and intrinsic birefringence, liquid crystals (LCs) are particularly well suited to these studies because the evolution of domains can be monitored in situ through polarizing optical microscopy on practical spatial and temporal scales [13-15].

LCs are anisotropic fluids that combine the fluidity of isotropic liquids as well as the long-range order of crystalline solids [16]. They are characterized by certain symmetries in the preferred orientations of LC molecules, i.e. the director $\mathbf{n}$ [16]. So far, most experimental investigations have been focused on the coarsening of topological defects induced by a drastic phase transition of LCs from the isotropic to the nematic phase [17-22]. Such a phase transition can induce different kinds of topological defects which vary from $\pi_{1}$ to $\pi_{3}$ homotopy classes of the vacuum manifold. However, different types of defects behave different scaling laws [23, 24], which may greatly reduce the reliability and accuracy of measurements.

In this paper, the attention is focused on the so-called reverse tilt domains (RTDs) in a nematic LC. RTDs are domains separated by walls [25]. In adjacent domains $\mathbf{n}$ tilts into two different orientations [26-28]. Due to the minimization of the free energy of the system, these RTDs are transient and will continuously shrink and eventually annihilate. They are favourable for the investigation of coarsening phenomenon because their configurations are very well defined with the shape of "closed loops". However, so far, the annihilation dynamics of RTDs has been rarely investigated [29], with no dependence on external field parameters reported. This may be because of the fact that RTDs are usually inhibited by the pretilt angle of the alignment layers in most LC devices. Here, to obtain an adequate number of RTDs, a process called photo-alignment technique is applied [30-32], which can provide an alignment condition with nearly zero pretilt angle. We investigate in detail the coarsening phenomenon as well as the annihilation dynamics of single RTDs. In addition, the effects of electric field amplitude, $E$, frequency, $f$, cell gap, $d$, and temperature, $T$, on the annihilation dynamics of RTDs are also investigated.

\section{Experimental section}

Materials. The nematic LC used is a standard commercially available material, E7 (Xianhua, China), with a positive dielectric anisotropy of $\Delta \varepsilon \sim 12$ and a positive conductivity anisotropy of $\Delta \sigma \sim 10^{-7} \Omega^{-1} \mathrm{~m}^{-1}$ [33]. The nematic is heated to the isotropic phase and filled into cells by capillary action.

Cell preparation. The cells for photo-alignment are made with two ITO coated glass substrates. The substrates were cleaned in an ultrasonic bath, plasma cleaned and spin-coated with a 0.1 wt \% solution of sulfonic azo dye (SD1) in dimethylformamide (DMF) [33, 34]. They were assembled using spacers with different thicknesses. The thicknesses of the cells, $d$, are measured by the thin film interference method [35], and were $d_{1}$ $=11.8 \mu \mathrm{m}, d_{2}=22.5 \mu \mathrm{m}$, and $d_{3}=36.8 \mu \mathrm{m}$, respectively. The empty cells were then exposed to linearly polarized ultraviolet (UV) light of wavelength $\lambda=395 \mathrm{~nm}$.

Generation of RTDs. An AC field with amplitude, $E$, and frequency, $f$, was the applied across samples by a waveform generator (33220A, Agilent) and a home-built power amplifier. For the temperature dependent investigations, the sample was heated to specific temperatures by a hot stage (LTSE350, Linkam) controlled by a temperature controller (TP 94, Linkam) with a relative accuracy of $0.1{ }^{\circ} \mathrm{C}$.

Optical characterization and measurements. Samples were observed through a polarizing optical microscope (POM) (Leica OPTIPOL) and the annihilation processes were recorded as 
movies by a digital camera (UI-3360CP-C-HQ, uEye Gigabit Ethernet) with a frame rate of $\sim 14.5 \mathrm{fps}$ and a resolution of $2032 * 1080$ pixels. The movies were then analyzed via open source software Image-J.

\section{Results and discussions}

\subsection{The annihilation dynamics of single isolated RTDs}
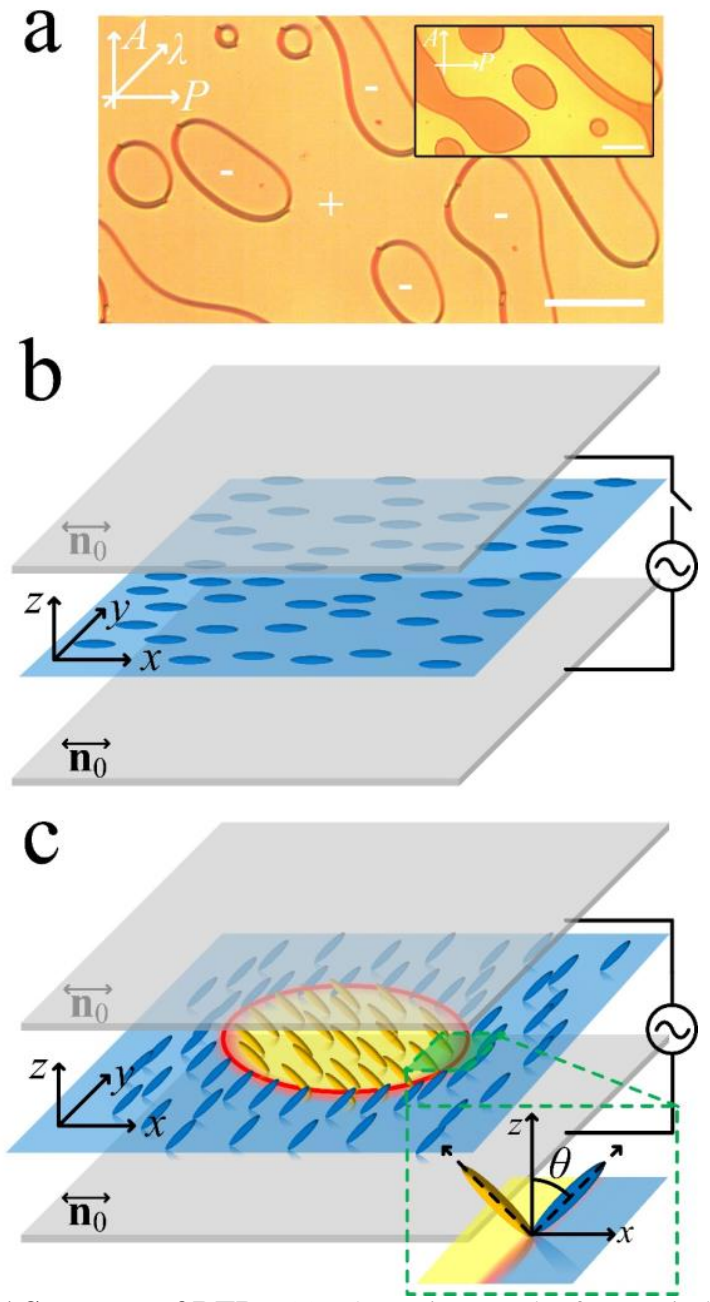

Figure 1 Structure of RTDs. (a) POM micrograph of RTDs induced by an AC field with amplitude $E=0.339 \mathrm{~V} \mathrm{\mu m}^{-1}$, frequency $f=1 \mathrm{KHz}$ The inset shows the texture of RTDs observed when the sample is tilted from the horizontal plane by $\sim 5^{\circ} . A, P$, and $\lambda$ indicate the analyzer, polarizer and red plate, respectively. (+) and (-) indicate different domains. Scale bars are $100 \mu \mathrm{m}$. (b) The schematic configuration of the sample before application of AC field. (c) The schematic configuration of different RTDs induced by applying AC field. The domain wall is illustrated by the red line. $\mathbf{n}_{0}$ indicates the alignment direction which is parallel to the $\mathrm{x}$-axis.

Figure 1 shows the texture and configuration of RTDs. When an electric field above the Freederickz threshold is applied perpendicular to the cell surfaces ( $x y$ plane), the middle-layer $\mathbf{n}$ tilts either clockwise or anticlockwise about the $y$-axis by a polar angle $\theta$ with respect to the $z$-axis (Figure 1c), with its azimuthal direction unchanged (along the $x$-axis). To confirm such a configuration, a first-order red plate compensator $(530 \mathrm{~nm})$ is inserted between the sample and the analyzer, with its slow axis $\lambda$ making $45^{\circ}$ with the analyzer. Neither a subtractive nor an additive effect is observed (Figure 1a), which demonstrates that there is no azimuthal deviation of $\mathbf{n}$ between the domains [36]. However, by tilting the sample with respect to the $x y$ plane by a small angle $\sim 5^{\circ}$, different colors of the domains can be distinguished (Figure 1a inset). Such a difference is induced by the different polar angle of $\mathbf{n}$ in different domains. The two polar directions of $\mathbf{n}$ are approximately degenerate in energy, and thus break the system into two states, (+) and (-) RTDs (Figure 1a), respectively. The boundaries of different domains emerge in the shape of "closed loops". To minimize the free energy of the system, these RTDs shrink and coarsen into a uniform director field over a time scale of seconds to minutes. We will refer to the boundaries of different RTDs (i.e. the domain walls) as "loops" from here on throughout the following text.
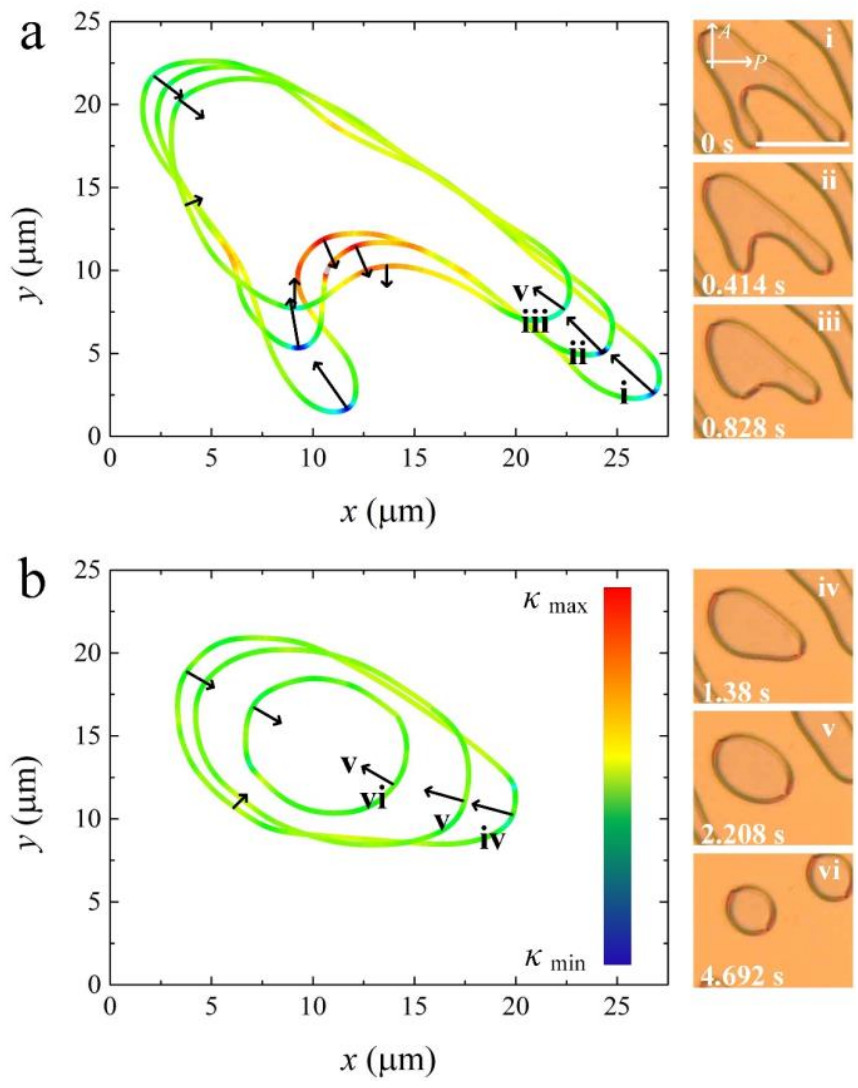

Figure 2 Shape evolution of a single RTD at different moments and the corresponding POM micrographs. (a) At the early stage of the annihilation where the loop changes its shape drastically. (b) at the late stage of the annihilation where the loop shrinks slowly with its shape unchanged. Black arrows indicate the velocities of different segments of the loop. The color of the loop indicates the local curvature. The color bar gives a linear scale of curvature $\kappa . \kappa_{\min }=-$ $1.24 \mu \mathrm{m}^{-1}$ and $\kappa_{\max }=0.49 \mu \mathrm{m}^{-1} . E=0.508 \mathrm{~V} \mathrm{\mu m}^{-1}, f=1 \mathrm{KHz}, d=11.8$ $\mu \mathrm{m}$. Scale bar $50 \mu \mathrm{m}$. $P$ and $A$ indicate the polarizer and analyzer, respectively.

Figure 2 shows the dynamical evolution of a single loop in the annihilation process. According to the Grayson theorem [37], any simple (not crossed) closed curve that moves under its curvature will shrink to a round shape regardless of its initial shape. In Figure $2 \mathrm{a}$, it is observed that the loop evolves quickly from an irregular shape into a circular one at the early stage of the annihilation. It is also observed that different segments of the loop move with different velocities, $\mathbf{v}$. The direction of $\mathbf{v}$ is along the normal direction of the segment and its amplitude is 
proportional to the local curvature, $\kappa$. Segments with curvatures of opposite signs move in opposite directions; and those whose curvatures are nearly zero almost do not move at all. At the late stage of the annihilation, the loop keeps its shape constant and slowly shrinks. At this stage, $\kappa$ is almost constant all over the loop (Figure 2b).
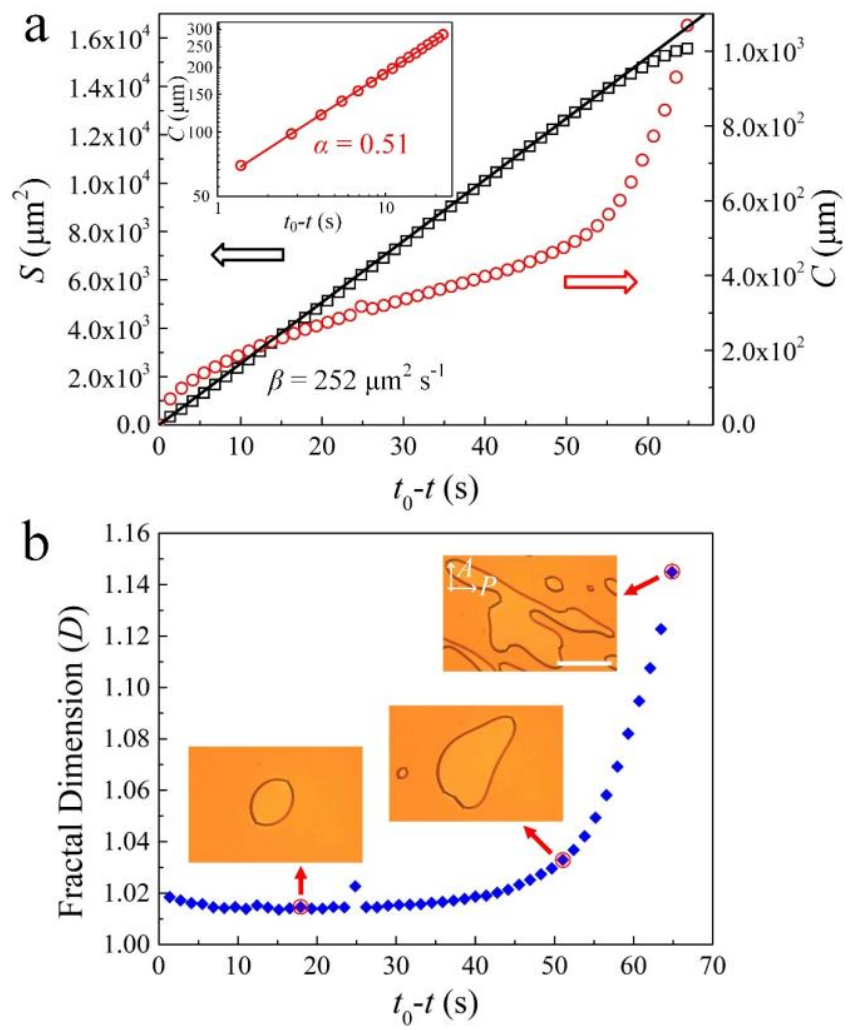

Figure 3 Exemplary annihilation dynamics of a single RTD. (a) Time dependence of the area, $S$, and the perimeter, $C$, of a single loop. $t_{0}-t$ is the time to annihilation. The inset gives the log-log plot of the perimeter vs time. (b) The dependence of the fractal dimension $D$ on time. The insets illustrate the POM textures of the loop at different moments indicated by the red arrows. $E=0.339 \mathrm{~V} \mu \mathrm{m}^{-1}, f=1 \mathrm{KHz}, d$ $=11.8 \mu \mathrm{m}$. Scale bar $100 \mu \mathrm{m} . P$ and $A$ indicate the polarizer and analyzer, respectively.

The time dependences of the areas, $S(t)$, as well as the perimeters, $C(t)$, of different loops was measured. One exemplary result is showed in Figure 3. One can clearly see that there is a drastic decrease of the perimeter at the early stage of the annihilation $\left(t_{0}\right.$ $-t>\sim 50 \mathrm{~s}$ ). This decrease is corresponding to the rapid shape transformation of the loop mentioned above. After the transformation, the perimeter shows a time dependence that can be expressed by

$$
C(t) \propto\left(t_{0}-t\right)^{\alpha}
$$

with $t_{0}-t$ the time to annihilation and $\alpha=0.51$. This dependence is in accordance to the scaling law of the annihilation of type $\pm 1 / 2$ disclination loops in isotropic-nematic phase transitions reported before[20,23,24], where $\alpha=0.5$. This scaling dynamics can be understood from the competition between the tension and the viscous drag force. We assume that at the late stage of the annihilation, the loop has a constant tension $T$ and a constant mobility $\Gamma . R$ denote the local radius of curvature, and the dynamics is described by [23]

$$
\frac{T}{R}=-\Gamma \frac{d R}{d t}
$$

By integrating, yields

$$
R=\sqrt{\frac{2 T}{\Gamma}\left(t_{0}-t\right)}
$$

On the other hand, surprisingly, the area of the loop shows a nearly linear dependence on time in spite of the rapid shape transformation of the loop at the early stage. The slope of the dependence is $\beta=252 \mu \mathrm{m}^{2} \mathrm{~s}^{-1}$ in the present case. To study the dynamical self-similarity of the loop during annihilation, we investigated the time dependence of its fractal dimension, $D(t)$, which is defined by

$$
C^{1 / D(t)}(t)=q S^{1 / 2}(t)
$$

where $q=2 \pi^{1 / 2}$ is a constant independent of time [38]. The value of $D$ as compared to unity implies how far a loop is differing from the shape of a circle. In Figure $3 b$, one can observe that $D$ decreases drastically from $\sim 1.15$ to $\sim 1.02$ at the early stage of the annihilation process, $\left(t_{0}-t>\sim 50 \mathrm{~s}\right)$, which demonstrates the rapid transformation of the loop from an irregular shape to a circular one. After that, $D$ remains nearly constant with $t$, indicating the dynamical self-similarity of the shrinkage at the late stage.



Figure 4 The dependence of the fractal dimension $D$ at the late stage of the annihilation on the amplitude of the electric field $E$. The insets give the corresponding POM texture of the loops. $f=1 \mathrm{KHz}$, $d=11.8 \mu \mathrm{m}$. Scale bar $40 \mu \mathrm{m}$. White arrows indicate the polarizer and analyser, respectively. The black and red squares in the inset corresponding to $E=0.339 \mathrm{~V} \mathrm{~mm}^{-1}$ indicate the bend and twist distortion regions, respectively. The error bars are calculated from the standard deviation of measurements of five different loops at each electric field.

Figure 4 shows the dependence of $D$ at the late stage of annihilation on the amplitude of the applied electric field, $E$. One finds that $D$ decreases gradually by increasing $E$, which demonstrates that the larger the applied electric field amplitude $E$, the more circular is the loop (insets). This is because a large electric field induces a quasi-homeotropic state which reduces the elastic anisotropy between bend (black square, inset) and twist (red square, inset) distortions of $\mathbf{n}$. It should be noted that one may argue that this Freedericksz effect is dependent on the voltage rather than $E$. However, this is not exactly true in our case due to the relatively weak anchoring strength of the SD1 
orientation layer [33]. To make a clear conclusion, further investigations are required which are out of the scope of this text. In addition, one can also see from the insets that the thickness of the loops also decreases for increasing $E$ which can be attributed to the change of the director structure of the loop as shown in Figure 5a. Further increase of $E$ will induce the so-called "pincement" [25].

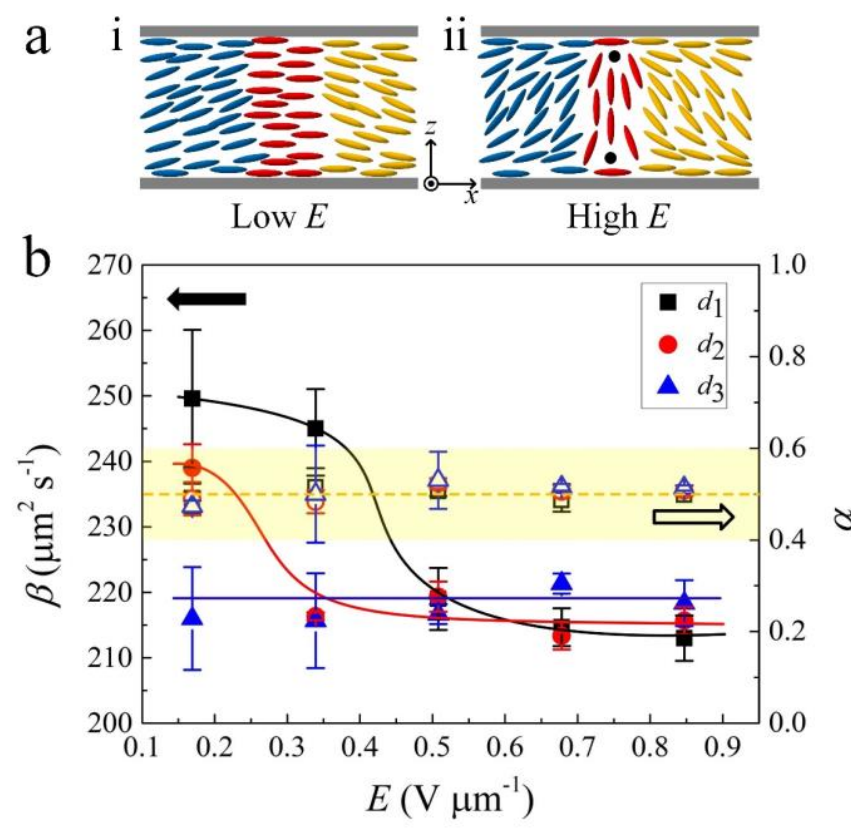

Figure 5 Electric field dependence of the RTDs. (a) Director configurations of the loops in the same cell at low (i) and high (ii) electric fields, respectively. (b) The dependences of $\alpha$ and $\beta$ of samples with different cell gaps $d$ on the amplitude of the electric field E. $f=1 \mathrm{KHz} . d_{1}=11.8 \mu \mathrm{m}, d_{2}=22.5 \mu \mathrm{m}$, and $d_{3}=36.8 \mu \mathrm{m}$, respectively. The error bars are calculated from the standard deviation of measurements of five different loops at each electric field.

The dependences of the annihilation dynamics of single loops on $E$ and $d$ were determined as depicted in Figure 5b. The annihilation exponent $\alpha$ is in the vicinity of $\alpha=0.5$ and is independent of both electric field $E$ and cell gap $d$. On the other hand, the coefficients $\beta$ of samples with a thicknesses of $d_{1}$ and $d_{2}$ decrease gradually for increasing $E$ until levelling at a constant value of $\beta \approx 220 \mu \mathrm{m}^{2} \mathrm{~s}^{-1}$. Such a decrease may be attributed to the transformation of the structure of the loops from configuration $i$ to configuration ii as shown in Figure 5a, which results in the change of tension of the loops. Another possible explanation is backflow effects which are dependent on the structure of $\mathbf{n}[15$, 39]. However, on the other hand, $\beta$ of the sample of $d_{3}$ shows independency of $E$. This may be due to the fact that the large cell gap reduces the elastic deformation energy and the confinement effect of the surface boundaries [40]. As a result, the loops are already in the configuration ii at low $E$.

Furthermore, the influences of the frequency of the electric field, $f$, and temperature $T$ on the annihilation dynamics of single RTDs were also investigated with results shown in Figure 6. It is observed that, as for the dependency on $E, \alpha$ remains constant in the vicinity of $\alpha=0.5$ and is independent on both $f$ and $T$. On the other hand, $\beta$ is independent on $f$ only, and shows a gradual increase with increasing $T$. Such an increase can be attributed to the decrease of the viscosity of the nematic LC for increasing temperature $[15,40]$.
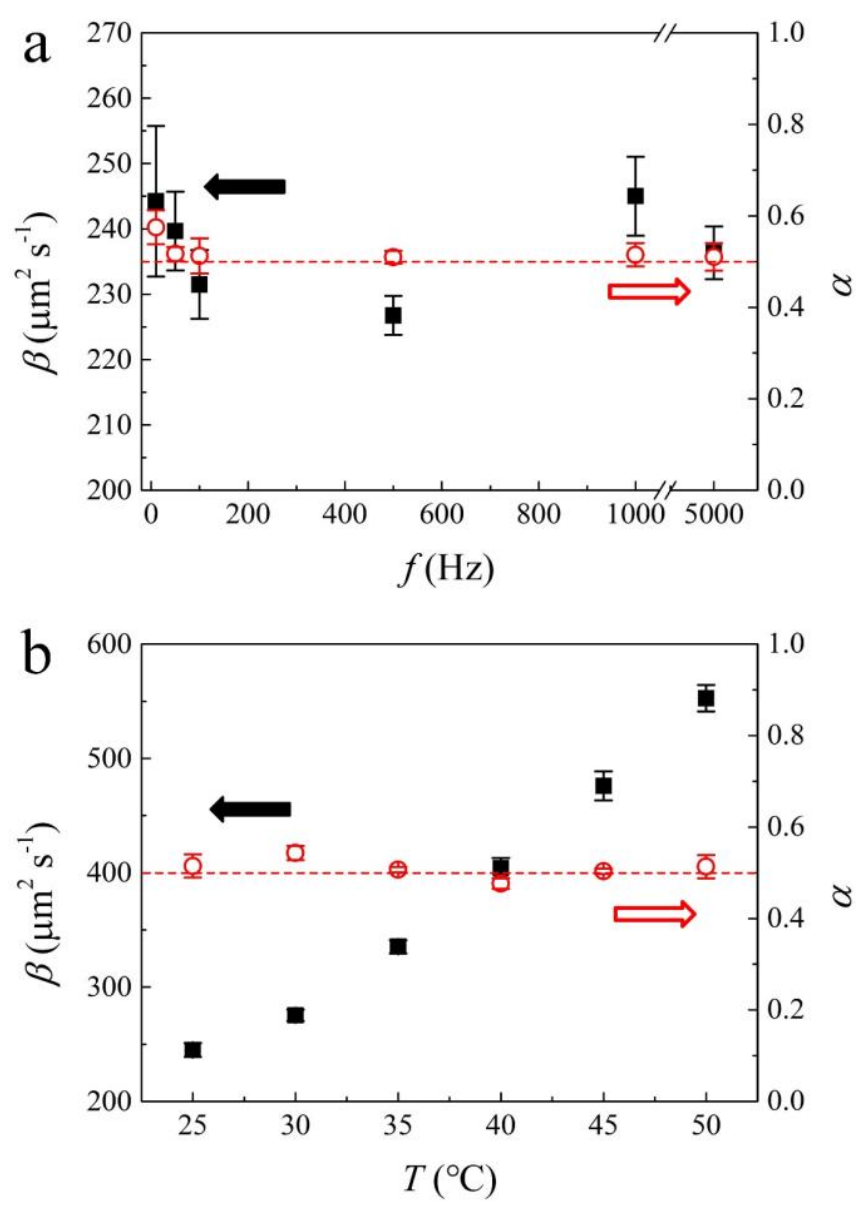

Figure 6 Frequency and Temperature dependences of the annihilation dynamics of RTDs. (a) The dependences of $\alpha$ and $\beta$ on

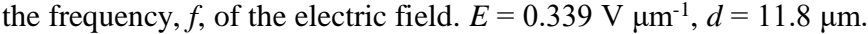
(b) The dependences of $\alpha$ and $\beta$ on the temperature, $E=0.339 \mathrm{~V} \mathrm{\mu m}^{-}$ $1, f=1 \mathrm{KHz}, d=11.8 \mu \mathrm{m}$. The red dashed lines indicate $\alpha=0.5$. The error bars are calculated from the standard deviation of measurements of five different loops at each frequency or temperature.

\subsection{Coarsening of RTDs}

The coarsening dynamics of a large number of RTDs was investigated as follows. Figure 7 depicts four micrographs showing the time evolution of the coarsening process. The images are processed with Image-J. The loops are shown in white and the interiors of both (+) and (-) domains are black. The number of white pixels in each image are measured as the length of all the loops, which is then divided by the number of all the pixels of the image to get $P$. In this sense, $P$ can be regarded as the total length of loops per unit area, $\rho$. The dependence of $P$ on time $t$ is shown in Figure 8a. One can see that $P$ decreases drastically at the initial stage and then remains nearly constant at the late stage of coarsening. Theoretically, this coarsening dynamics can be described by the "one-scale" model [13], in which the domain network can be characterized by a single length scale $L(t)$ which scales with time $t$ as $L(t) \propto t^{1 / 2[41-44]}$. In our case, the RTD system maps onto a two dimensional Ising model in which the (+) and (-) states corresponding to the Ising spin states $\sigma_{\mathrm{i}}= \pm 1$, and $\rho$ should scale as $\rho \propto L^{-1}[29,45]$. As a result, $\rho \propto t^{-1 / 2}[20]$. 

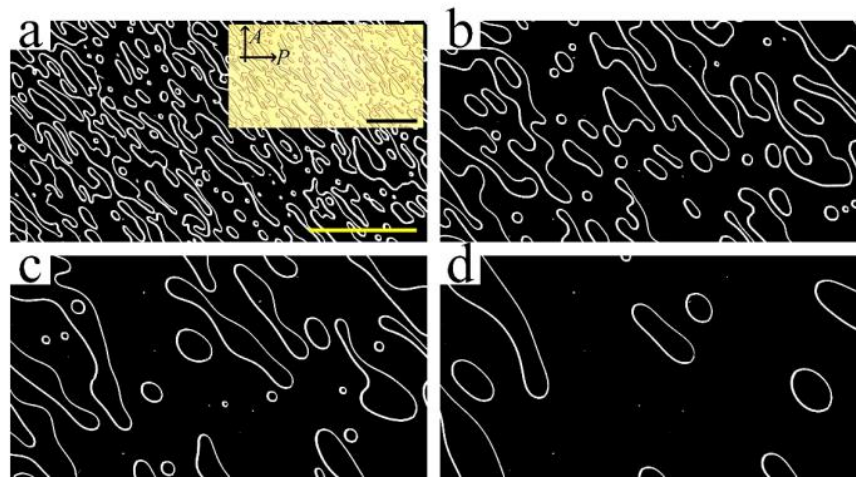

Figure 7 Micrographs of coarsening of loops at different moments processed by Image-J. (a) $t=0.276 \mathrm{~s}$. The inset gives the corresponding unprocessed POM micrograph. $P$ and $A$ indicate the polarizer and analyzer, respectively. (b) $t=1.518 \mathrm{~s}$. (c) $t=4.554 \mathrm{~s}$. (d)

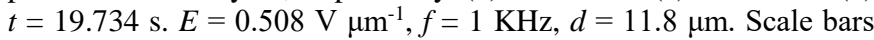
$200 \mu \mathrm{m}$.

However, according to ref [29], the heterogeneity of the surface is important to the formation of RTDs and may change the scaling law of coarsening.

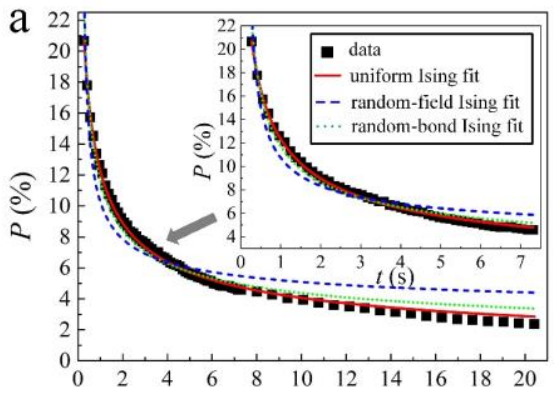

$t(\mathrm{~s})$

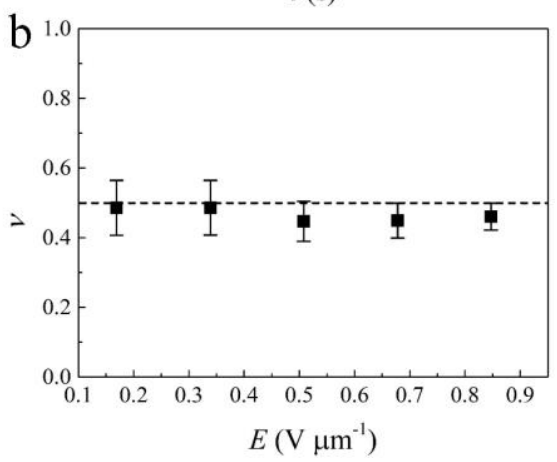

Figure 8 The dynamics of the coarsening of RTDs. (a) Data for the time dependence of the total perimeter of all the loops in the field of view. The data are fitted with the uniform Ising model (red solid line), the random-field Ising model (blue dashed line), and the random-bond Ising model (green dotted line), respectively. The inset gives the corresponding coarsening from $t=0 \mathrm{~s}$ to $t=7.5 \mathrm{~s}$. (b) The dependence of the coarsening exponent $v$ on the amplitude, $E$, of the electric field. The black dashed line indicates $v=0.5$. The error bars are calculated from the standard deviation of measurements of five different coarsening processes at each $E$.

We thus fit $P$ to the following functions as reported in ref [29]: (a) the uniform Ising model where the coarsening is controlled by the competition between the characteristic loop tension and the viscosity drag force [44]

$$
P(t)=c(t-\tau)^{-1 / 2}
$$

(b) the random-field Ising model where the coarsening is limited by the pinning forces related to the heterogeneity in the random field

$$
P(t)=c[\log (t / \tau)]^{-1}
$$

and (c) the random-bond Ising model where the coarsening is limited by the pinning forces related to the heterogeneity in the random bonds

$$
P(t)=c[\log (t / \tau)]^{-4}
$$

where $c$ is a coefficient and $\tau$ is the characteristic time scale.
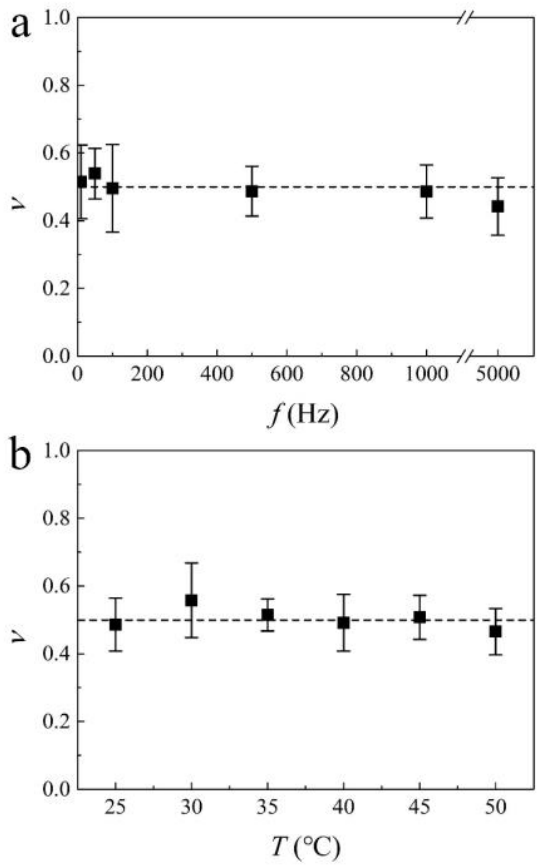

Figure 9 The dependence of coarsening of RTDs on external parameters. (a) The dependence of the coarsening exponent $v$ on the frequency, $f$, of the electric field. (b) the dependence of $v$ on temperature, $T$. The black dashed line indicates $v=0.5$. The error bars are calculated from the standard deviation of measurements of five different coarsening processes at each frequency or temperature.

Unlike the results reported in ref [29], where $\rho \propto(\log t)^{-4}$, it is found that the coarsening maps onto the uniform Ising model, and $\rho$ scales with time $t$ as

$$
\rho=c(t-\tau)^{-v}
$$

where $v=1 / 2$, as shown in Figure 8a. This may be due to the surface boundary in our case being relatively uniform without significant heterogeneity, which is exactly one of the advantages of the photo-alignment technique. However, on the other hand, in ref [29], the alignment was induced through chemisorption and photoinduced dimerization, and brittle glass spacers were introduced on purpose to induce an adequate number of RTDs. It is found that all the models start breaking down at longer time scales which may be attributed to finite size effects [24, 29]. Furthermore, the dependences of the coarsening exponent $v$ on $E, f$, and $T$ are also investigated in Figure $8 \mathrm{~b}$ and Figure 9, respectively, in which $v$ is in the vicinity of $v=1 / 2$ with no distinct dependency. 


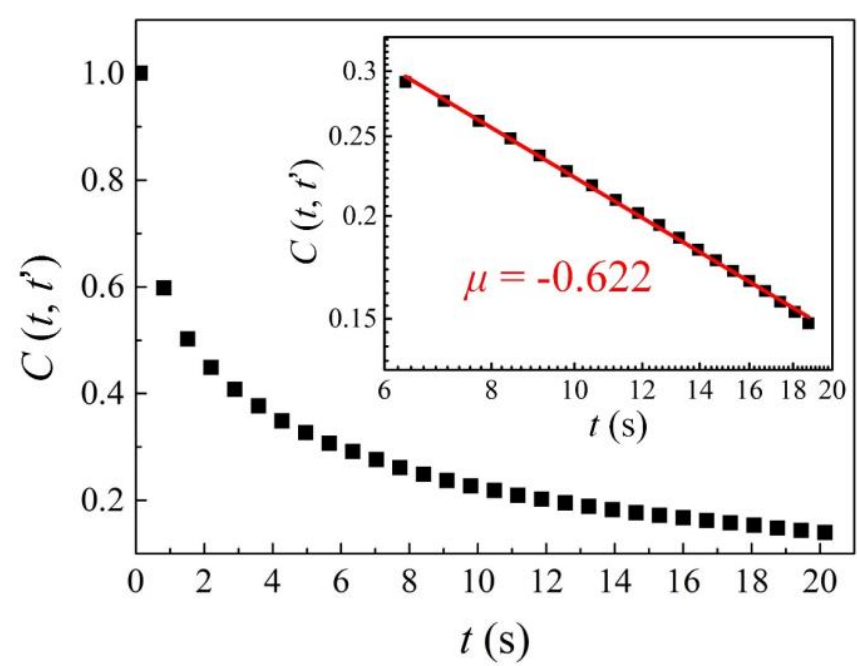

Figure 10 Exemplary dependence of the two-time correlation function, $\boldsymbol{C}\left(t, t^{\prime}\right)$, on time, $t$. The inset shows the log-log plot of the dependence from $t \sim 6 \mathrm{~s}$ to $t \sim 19 \mathrm{~s}$. The red solid line has a slope of $\mu$ $=-0.622$.

Finally, we investigated the exponent characterizing the asymptotic behaviour of the two-time correlation function [46]

$$
\begin{aligned}
& C\left(t, t^{\prime}\right)=\left\langle\Phi(r, t) \Phi\left(r, t^{\prime}\right)\right\rangle \\
& =(1 / N) \sum_{j} \Phi_{j}(t) \Phi_{j}\left(t^{\prime}\right)
\end{aligned}
$$

where $t^{\prime}$ is selected to be the moment right after the application of electric field, which is $\sim 0.14 \mathrm{~s}$, the sum of pixels is $N=$ $2032 * 1080$, and the order parameters $\Phi_{j}(t)$ take the values +1 and -1 , depending on whether the pixel corresponds to the (+) or (-) region (Figure 1a). When $t>>t^{\prime}$, the correlation function scales with the length scale $L(t)$ as $C\left(t, t^{\prime}\right) \propto L(t)^{\xi}$, where $\xi$ is expected to be -1.25 for an ideal two dimensional Ising system [46, 47]. In Figure 10, It is found that $C\left(t, t^{\prime}\right)$ scales with time $t$ from $\sim 6 \mathrm{~s}$ to $\sim 19 \mathrm{~s}$ with an exponent $\mu=-0.622$. We measured five different coarsening runs, and obtained an averaged exponent $[\mu]=0.633$. Since $C\left(t, t^{\prime}\right) \propto t^{\mu}$, and $L(t) \propto t^{v}, \lambda$ can thus be given by $\xi=$ $\mu / v$. The average value of $v$ at the same conditions of $[\mu](T=25$ ${ }^{\circ} \mathrm{C}, d=11.8 \mu \mathrm{m}, E=0.339 \mathrm{~V} \mu \mathrm{m}^{-1}, f=1 \mathrm{KHz}$ ) is given in Figure $8 \mathrm{~b},[v]=0.486$. Thus the average value of $\xi$ can be given by $[\xi]$ $=[\mu] /[v]=-1.302$, which is close to the expected value of -1.25 .

\section{Conclusions}

The annihilation dynamics of single RTDs was investigated. The motion of domain walls is driven by the competition between the curvature induced force and the viscous drag force, which gives a scaling law of equation (1). The annihilation exponent was found as $\alpha=1 / 2$ and independent of external parameters, including electric field, temperature, and cell gap, demonstrating its universality. The coarsening dynamics of RTDs maps onto the uniform Ising model and presents a scaling law of equation (8) with an exponent $v=1 / 2$ which is also independent on external parameters.

\section{Conflicts of interest}

The authors declare no competing interests.

\section{Acknowledgements}

The authors would like to acknowledge Prof. Lujian Chen for supplying SD1 and Adam Draude for help with plasma cleaning ITO glass and spin-coating SD1. Y.S. gratefully acknowledges the China Scholarship Council (CSC) for kind support under grant number 201806310129.

\section{Notes and references}

[1] T.W. Kibble, Journal of Physics A: Mathematical and General 9 (1976) 1387.

[2] W.H. Zurek, Nature 317 (1985) 505.

[3] M.J. Bowick, L. Chandar, E.A. Schiff, A.M. Srivastava, Science 263 (1994) 943.

[4] P.W. Voorhees, Journal of Statistical Physics 38 (1985) 231.

[5] H. Toyoki, K. Honda, Progress of Theoretical Physics 78 (1987) 237.

[6] M. Salomaa, G. Volovik, Reviews of modern physics 59 (1987) 533.

[7] R. Durrer, New astronomy reviews 43 (1999) 111.

[8] M.K. Phani, J.L. Lebowitz, M.H. Kalos, O. Penrose, Physical Review Letters 45 (1980) 366.

[9] T. Ohta, D. Jasnow, K. Kawasaki, Physical Review Letters 49 (1982) 1223.

[10] H. Furukawa, Advances in physics 34 (1985) 703.

[11] A. Sicilia, J.J. Arenzon, I. Dierking, A.J. Bray, L.F. Cugliandolo, J. Martinez-Perdiguero, I. Alonso, I.C. Pintre, Physical review letters 101 (2008) 197801.

[12] A. Bray, Physical review letters 62 (1989) 2841.

[13] I. Chuang, R. Durrer, N. Turok, B. Yurke, Science 251 (1991) 1336.

[14] I. Dierking, O. Marshall, J. Wright, N. Bulleid, Physical Review E 71 (2005) 061709.

[15] Y. Shen, I. Dierking, Soft Matter 15 (2019) 8749.

[16] Y. Shen, I. Dierking, Applied Sciences 9 (2019) 2512.

[17] I. Chuang, N. Turok, B. Yurke, Physical Review Letters 66 (1991) 2472.

[18] R. Snyder, A. Pargellis, P. Graham, B. Yurke, Physical Review A 45 (1992) R2169.

[19] A.P. Wong, P. Wiltzius, B. Yurke, Physical review letters 68 (1992) 3583.

[20] H. Orihara, Y. Ishibashi, Journal of the physical society of Japan 55 (1986) 2151.

[21] T. Nagaya, H. Orihara, Y. Ishibashi, Journal of the Physical Society of Japan 56 (1987) 3086.

[22] T. Nagaya, H. Orihara, Y. Ishibashi, Journal of the Physical Society of Japan 56 (1987) 1898.

[23] B. Yurke, A.N. Pargellis, I. Chuang, N. Turok, Physica B: Condensed Matter 178 (1992) 56.

[24] I. Chuang, B. Yurke, A.N. Pargellis, N. Turok, Physical Review E 47 (1993) 3343.

[25] P.-G. De Gennes, J. Prost, The physics of liquid crystals (2nd Edition). Oxford university press, 1993.

[26] S.H. Lee, T.-H. Yoon, J.C. Kim, G.-D. Lee, Journal of Applied Physics 100 (2006) 064902.

[27] S.-K. Kim, J.-H. Lee, Japanese Journal of Applied Physics 50 (2011) 050203.

[28] L. Léger, Molecular Crystals and Liquid Crystals 24 (1973) 33.

[29] D.K. Shenoy, J.V. Selinger, K.A. Grüneberg, J. Naciri, R. Shashidhar, Physical Review Letters 82 (1999) 1716. 
[30] S.-S. Li, Y. Shen, Z.-N. Chang, W.-S. Li, Y.-C. Xu, X.-Y. Fan, L.-J. Chen, Applied Physics Letters 111 (2017) 231109.

[31] Y. Shen, Y.-C. Xu, Y.-H. Ge, R.-g. Jiang, X.-Z. Wang, S.-S. Li, L.-J. Chen, Opt. Express 26 (2018) 1422.

[32] Y. Shen, I. Dierking, Communications Physics 3 (2020) 1.

[33] Y. Shen, I. Dierking, Soft Matter (2020) DOI:10.1039/d0sm00676a.

[34] L.-L. Ma, S.-S. Li, W.-S. Li, W. Ji, B. Luo, Z.-G. Zheng, Z.-P. Cai, V. Chigrinov, Y.-Q. Lu, W. Hu, L.-J. Chen, Advanced Optical Materials 3 (2015) 1691.

[35] A.M. Goodman, Appl. Opt. 17 (1978) 2779.

[36] N.H. Hartshorne, The Microscopy of Liquid Crystals. Microscope Publications Ltd., 1974.

[37] J. Gunther, E.L. Thomas, S. Clingman, C.K. Ober, Polymer 39 (1998) 4497.

[38] W. Wang, T. Shiwaku, T. Hashimoto, The Journal of Chemical Physics 108 (1998) 1618.

[39] G. Tóth, C. Denniston, J.M. Yeomans, Physical Review E 67 (2003) 051705.

[40] I. Dierking, M. Ravnik, E. Lark, J. Healey, G. Alexander, J. Yeomans, Physical Review E 85 (2012) 021703.

[41] I.M. Lifshitz, V.V. Slyozov, Journal of physics and chemistry of solids 19 (1961) 35.

[42] O.D. Lavrentovich, P. Pasini, C. Zannoni, S. Zumer, Defects in liquid crystals: Computer simulations, theory and experiments. Springer Science \& Business Media, 2012.

[43] A.J. Bray, Advances in Physics 51 (2002) 481.

[44] S.M. Allen, J.W. Cahn, Acta Metallurgica 27 (1979) 1085.

[45] M. Lederman, J. Selinger, R. Bruinsma, R. Orbach, J. Hammann, Physical Review B 48 (1993) 3810.

[46] N. Mason, A.N. Pargellis, B. Yurke, Physical Review Letters 70 (1993) 190.

[47] D.S. Fisher, D.A. Huse, Physical Review B 38 (1988) 373. 